\title{
The effect of percutaneous partial release of the plantar fascia and prolotherapy in plantar fasciitis cases: Patient expectations and treatment effects
}

\author{
Zeki Taşdemir* \\ Department of Orthopedics and Traumatology, Health Sciences University Kartal Dr. Lütfi Kurdar Training and Research Hospital, İstanbul
}

\begin{abstract}
Introduction: Plantar fasciitis $(\mathrm{PF})$ is a common cause of heel pain and manifests itself as pain surrounding the calcaneal tubercle. The pain is more severe when one steps on the ground for the first time in the morning, and the plantar fascia decreases as it warms up. Plantar fasciitis can respond to conservative treatments such as ice, relaxation and anti-inflammatory agents. Limited interest has been shown in exercise-based therapies for this common problem.

This study aims to make a clinical contribution to the efficacy of plantar release and prolotherapy in the treatment of PF. Similarly, it was examined whether a detailed explanation of form of treatment had an effect on recovery.

Material and method: This study was designed as retrospective. Adults diagnosed with plantar fasciitis and followed-up for at least 3 months were included in the study. All patients followed the same exercise protocol and used the same anti-inflammatory agents. In their controls in Weeks 3 , 24 and 48 the severity of pain was assessed based on Visual Analogue Scale (VAS) for Pain. The patients were informed that some of them would be administered a prolotherapy injection at the end of the conservative treatment.

Findings: Groups that were administered prolotherapy and followed-up conservatively reported that their pain significantly alleviated in the $48^{\text {th }}$ week. In the $24^{\text {th }}$ week controls, it was determined that the pain scores of prolotherapy group significantly decreased in patients to whom prolotherapy was administered compared to the conservative group.

Conclusion: The exercise regime applied in this study alleviates the pain associated with chronic plantar facitiis. However, prolotherapy resulted in a decrease in pain in the early period compared to the conservative treatment. Pre-treatment (prolotherapy) VAS scores of patients who were informed about what would be done in the post-treatment were lower than the patients who were not informed.
\end{abstract}

\section{Introduction}

The term "tendinosis", which is a more degenerative description, is preferred for PF instead of tendinitis or enthesitis that reflects an active inflammatory condition. A chronic inflammation lies beneath the PF that occurs due to various causes. Instead of tendinitis or enthesitis that reflects an active inflammatory state for PF, tendinosis is preferred, which is a more degenerative description [1].

Prolotherapy is used for the treatment of insufficiently recovered structures. It is a treatment method that involves repeated injections based on the injection of proliferative substances into ligaments and tendons [2]. It is used in the treatment of chronic problems such as enthesitis and tendinosis where degeneration underlies [3]. Plantar fasciitis relaxation is applied in PFs that do not recover.

Literature does not contain comparative information on available evidence for prolactotherapy or conservative treatment. There is literature on the available evidence to manage patient expectations and its impact on outcomes.

Psychological interventions for patients with chronic pain may help patients feel better in pain control and may lead to a normal life as much as possible despite pain. In addition, the skills acquired through psychological interventions enable patients to become active participants in the management of their illnesses and undergo valuable skills that patients can use throughout their lives [4].

In this study, partial plantar release and prolotherapy are coadministered. Thus, we believe that PF pain will be treated faster and permanently. The aim was to investigate the efficiency of both treatment methods and make clinical contributions. Similarly, it was examined whether detailed explanation of route of treatment had an effect on recovery.

\section{Materials and methods}

A total of 104 patients who applied to orthopaedics polyclinic of our hospital and diagnosed with plantar fasciitis and administered

${ }^{*}$ Correspondence to: Zeki Taşdemir, Department of Orthopedics and Traumatology, Health Sciences University Kartal Dr. Lütfi Kırdar Training and Research Hospital, İstanbul, E-mail: drtazeki@gmail.com

Key words: conservative treatment, plantar fasciitis, prolotherapy

Received: April 12, 2019; Accepted: April 25, 2019; Published: April 29, 2019 
prospectively cohort prolotherapy and followed-up conservatively were screened. This study was started with the permission of Kartal Education and Research Hospital Ethics Committee of 20017/514/1000/8 and dated 31.01.2017. The diagnosis of plantar fasciitis was made by clinical and USG showing the inflammation in the plantar fascia. Patients, who had plantar fasciitis complaints for at least 12 weeks and were not subject to any procedure other than the medical treatment were included in the study. 9 patients who had a history of foot and ankle injuries and operation and rheumatic disease and foot neuropathy (diabetic neuropathy) were excluded from the study. 6 patients who do not want to participate voluntarily in the study and 16 patients who do not come to the follow-up or use cushioned heel shoes and do not administer the proper treatment were excluded from the study. 73 patients were included in our study. Patients with bilateral plantar fasciitis were evaluated for the most painful side.

All data of the patients were processed in the patient record system (octomed) and accessed. Patients who were informed about prolotherapy before conservative treatment were told that $10 \%$ dextrose should be applied to the painful area 10 times after the conservative treatment for 3 weeks.

In our study, the severity of pain in the first step in the morning that is evaluated as diagnostic in patients with PF was evaluated based on VAS. Patients were instructed to mark the severity of their pain on a $100 \mathrm{~mm}$ chart in their controls at the time of application and week 24 and 48 . Thus, they could mark by making a comparison to the previous pain level. The decision to administer prolotherapy to patients and whether to inform them about the treatments at the beginning was taken randomly. Evaluations were based on the most painful side of patients diagnosed with plantar fasciitis on both feet.

All patients with plantar fasciitis who applied to our orthopaedics polyclinic started with plantar stretching exercises, cold application and Naproxen NA $100 \mathrm{mg} /$ day for 3 weeks. All patients were recommended standard orthopaedic footwear and slippers. Some patients were informed that prolotherapy could be administered after 3 weeks of conservative treatment. Other patients were not informed about prolotherapy. VAS was evaluated at the Week 3.

Patients who were administered prolotherapy were duly informed and their written consents were taken and prolotherapy was applied. In practice, the trigger point was searched at the base of the feet and injection was made to these points. For this purpose, the skin was wiped with an antiseptic solution. Painful spot was marked with palpation. The prepared $10 \%$ dextrose solution was applied to painful spots in the calcaneus adhesion region of the plantar fascia, the periosteum and thickened plantar fascia. Application was made from the same inlet for at least 15 times without pulling back the injector tip $(0.8 \times 38 \mathrm{~mm})$ by 5-10 $\mathrm{mm}$ withdrawal and drawing plantar fascia in different angles with the injector tip and pricking into calcaneal periosteum. Then, $40 \mathrm{cc} 10 \%$ dextrose was injected without pulling back the injector and the process was terminated.(Figure 1) Application was a single session without local anaesthesia Patients were informed that pain and rash might occur in injection sites. Injection was not reiterated in patient controls.

Stretching exercises and ice application was re-explained through brochure to the patient group to which conservative treatment was applied. Naproxen NA treatment (100 mg/day) was continued. VAS scores in the follow-ups were recorded as VAS1 in Week 3; VAS2 in Week 24 and VAS3 in Week 48.

Grouping: Patients who were administered prolotherapy were categorized into two groups as those informed about the prolotherapy

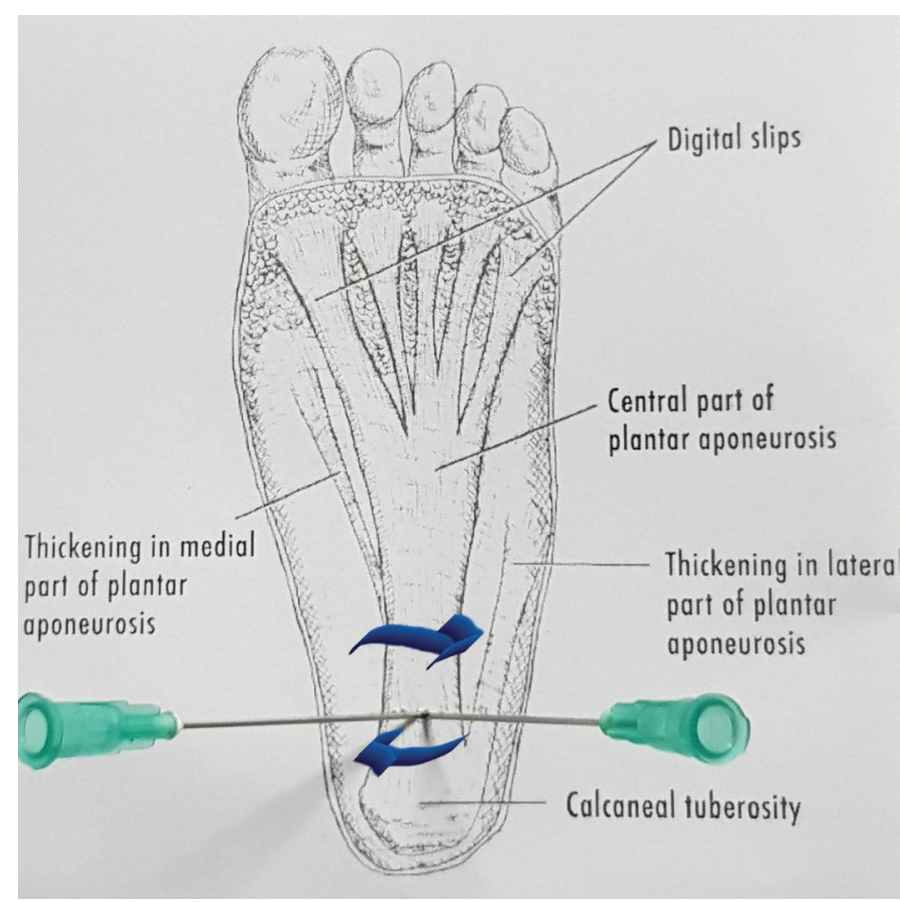

Figure 1. Multiple perforations opened by the tip of injector during prolotherapy result in partial release of plantar fascia

and those who were not. Similarly, patients with conservative treatment were categorized into two based on whether prolotherapy was described or not when they first came.

\section{Istatiksel analiz}

Independent Samples - Mann Whitney U test was used from among non-parametric tests to compare the VAS of patients who were administered prolotherapy and followed-up with conservative treatment. When our groups were small, powerful analysis was performed in the results.

\section{Findings}

All patients were ladies. 27 of the patients (37.0\%) had left-side pain; 31 of the patients $(42.5 \%)$ had right-side pain and 15 patients (20.5\%) had pain in both sides. 14 patients $(19.2 \%)$ had diabetes. Out of the patients included in the analysis, 29 patients (39.7\%) were informed that injection might be administered in the conservative post-treatment while 44 of the patients (60.3\%) were not informed.

In order to compare VAS of 19 patients with DM and 54 patients without DM, was no significant difference between the averages of all VAS scores. The age of the patients included in the analysis was $50.00 \pm$ 1.259 (32-61); their BMI scores were $26.11 \pm 0.45$ (24-33); their VAS1 scores were $65.93 \pm 1.89$; their VAS2 scores were $44.23 \pm 1.41$ and their VAS3 scores were $38.75 \pm 1.189$.

Compare the VAS scores of patients who were administered prolotherapy and followed-up with conservative treatment. According to the results of comparison of the VAS of the 28 patients who were injected with 45 patients who were not injected, no significant difference was found between the averages of VAS1 (before injection) scores whereas significant differences were found between VAS2 and VAS3 scores (Table 1). Power analysis results are given in Table 2.

Compare the VAS scores of patients who were administered prolotherapy and were explained in detail or not explained in detail on 
Table 1. VAS score relationship between patients with prolotherapy and patients followedup with conservative therapy

\begin{tabular}{|c|c|c|c|c|c|c|}
\hline & Proloterapy & $\mathrm{N}$ & Mean & Std. Deviation & $\begin{array}{c}\text { Std. Error } \\
\text { of Mean }\end{array}$ & $\mathrm{p}$ \\
\hline \multirow{2}{*}{ VAS1 } & 0 & 45 & 63,96 & 13,671 & 2,038 & \multirow{2}{*}{0.256} \\
\cline { 2 - 6 } & 0 & 28 & 69,11 & 19,477 & 3,681 & \\
\hline \multirow{2}{*}{ VAS2 } & 0 & 45 & 47,47 & 11,866 & 1,769 & \multirow{2}{*}{0.002} \\
\cline { 2 - 6 } & 1 & 28 & 39,04 & 10,675 & 2,017 & \\
\hline \multirow{2}{*}{ VAS3 } & 0 & 45 & 41,27 & 9,355 & 1,395 & \multirow{2}{*}{0.006} \\
\cline { 2 - 6 } & 1 & 28 & 34,71 & 10,255 & 1,938 & \\
\hline
\end{tabular}

Table 2. Power analysis; Compare the VAS scores of patients who were administered prolotherapy and followed-up with conservative treatment

\begin{tabular}{|l|c|c|c|c|c|c|}
\hline & Enjeksiyon & N & Mean & $\begin{array}{c}\text { Std. } \\
\text { Deviation }\end{array}$ & $\begin{array}{c}\text { Std. Error } \\
\text { of Mean }\end{array}$ & P Power \\
\hline \multirow{2}{*}{ VAS1 } & 0 & 45 & 63,96 & 13,671 & 2,038 & 0.256 \\
\cline { 2 - 7 } & 1 & 28 & 69,11 & 19,477 & 3,681 & 0.359 \\
\hline \multirow{2}{*}{ VAS2 } & 0 & 45 & 47,47 & 11,866 & 1,769 & 0.002 \\
\cline { 2 - 7 } & 1 & 28 & 39,04 & 10,675 & 2,017 & 0.906 \\
\hline \multirow{2}{*}{ VAS3 } & 0 & 45 & 41,27 & 9,355 & 1,395 & 0.006 \\
\cline { 2 - 6 } & 1 & 28 & 34,71 & 10,255 & 1,938 & 0.857 \\
\hline
\end{tabular}

their first arrival which treatments would be administered. According to the results of comparison of the VAS scores of the 16 patients who were informed with 12 patients who were not informed, a significant difference at a confidence level of $99 \%$ was found in the averages of VAS1 and VAS2 scores of the patients who were informed and were not informed about the prolotherapy. But any significant difference was not found between their average VAS3 scores (Table 3). The results of the Power analysis were performed in patients with prolotherapy. There is a significant difference in the power analysis at $99 \%$ confidence level between the averages of vas 1 scores. $(\mu D A 1=60.38, \operatorname{SEDA} 1=4.514$, $\mu \mathrm{DA} 0=80.75, \mathrm{SEDA} 0=4.357, \mathrm{p}=0.004)$. There is a significant difference between the mean of Vas2 scores and 99\% confidence level power analysis. $(\mu \mathrm{DA} 1=34.50, \mathrm{SEDA} 1=2.737, \mu \mathrm{DA} 0=45.08, \mathrm{SEDA} 0=1.967$, $\mathrm{p}=0.007)$.

Compare the VAS scores of patients who were followed up with conservative therapy and were explained and were not explained in detail on their first arrival which treatments would be administered. According to the results of the comparison of the VAS scores of the 17 patients who were informed and 28 patients who were not informed, a significant difference at the confidence level of $99 \%$ was found between patients who were informed and those who were not informed (Table 4). The results of the Power analysis were performed in patients without prolotherapy. There is a significant difference in the $99 \%$ confidence level between the averages of vas 1 scores. $(\mu \mathrm{DA} 1=58.54$, SEDA $1=2.535$, $\mu \mathrm{DA} 0=72.88, \mathrm{SEDA} 0=2.091, \mathrm{p}=0.000$ ). During the first and second days of injection, no complications were reported except local pain and tenderness.

\section{Discussion}

There are two approaches to the treatment of plantar fasciitis: conservative and surgical treatment. There is a significant consensus that conservative treatment will be sufficient for $70-90 \%$ of the patients with plantar fasciitis $[5,6]$. Methods used in the initial stage of the treatment protocol involve the regulation of rest and activities, ice application, stretching techniques, NSAI agents, heel pads and soles and weight [7-9]. Unless sufficient recovery is achieved in 6-8 weeks, injection treatments (steroid, dextrose, botulinum toxin, platelet-rich plasma (PRP) and dry needling are used [10-12]. ESWT and plantar fasciotomy are recommended in patients with persistent plantar fasciitis whose symptoms do not regress with conservative treatment for more than 6 months $[5,11]$.

In the study conducted by Martin JE et al., bandage and the results were checked in the follow-ups. Patients who failed to meet these criteria were excluded from the study. Mechanical treatment with orthotics was found to be more effective than anti-inflammatory or soothing modality and a statistically significant difference was found [11]. In our study, plantar fascia stretching exercises, anti-inflammatory treatment and simple soft footwear and slippers were recommended.

Injection treatments are commonly used in persistent plantar fascias and steroid injections are the most common application. In most studies, the efficacy analysis of the treatment method is compared with steroid injections $[7,10,11]$. In chronic pain of musculoskeletal system, prolotherapy that is widely used for around 80 years is increasingly used to treat plantar fasciitis as well $[13,14]$. Van Pelt defined prolotherapy injection sites in plantar fasciitis as heel, mid-arch and metatarsal heads and reported that the most painful area of the intervention was the heel and the most painless area was the mid-arch [12]. In our study, invasion was made with the tip of injector to the heal area that is the most painful area and the fibrotic region and thus, plantar relaxation was achieved. A single-dose prolotherapy was applied and the procedure was terminated.

Non-invasive treatment options are available in plantar fasciitis, but they are usually ineffective, which are followed by steroid infiltration or operative treatment. Our home education program is effective and useful for orthopaedics and general practice. Invasive treatment is not allowed in plantar fasciitis unless a long-term and fair conservative treatment is administered [15].

The efficacy of exercise in the treatment of PF: Exercises can be considered to be more important than prolotherapy in clinical recovery. As none of the groups was treated only with exercises, it is difficult to report this [16]. The group followed-up with conservative treatment in our study enabled the formation of the control group.

Hauser et al. reported in their study where prolotherapy was compared with PRP injection that long-term consequences were similarly effective in both methods [17]. In our study, VAS scores of

Table 3. The relationship of VAS between those who were informed and were not informed about prolotherapy in patients who were administered prolotherapy $(0=$ not informed, $1=$ informed)

\begin{tabular}{|c|c|c|c|c|c|c|}
\hline & İnformed & $\mathbf{N}$ & Mean & $\begin{array}{c}\text { Std. } \\
\text { Deviation }\end{array}$ & $\begin{array}{c}\text { Std. Error } \\
\text { of Mean }\end{array}$ & p \\
\hline \multirow{2}{*}{ VAS1 } & $0^{*}$ & 12 & 80,75 & 15,094 & 4,357 & \multirow{2}{*}{0.004} \\
\cline { 2 - 7 } & $1^{*}$ & 16 & 60,38 & 18,055 & 4,514 & \\
\hline \multirow{2}{*}{ VAS2 } & $0^{*}$ & 12 & 45,08 & 6,815 & 1,967 & \multirow{2}{*}{0.007} \\
\cline { 2 - 7 } & $1^{*}$ & 16 & 34,50 & 10,948 & 2,737 & \multirow{2}{*}{ VAS3 } \\
\cline { 1 - 7 } & $0^{*}$ & 12 & 36,08 & 9,219 & 2,661 & \multirow{2}{*}{$1^{*}$} \\
\cline { 2 - 7 } & 16 & 33,69 & 11,152 & 2,788 & \\
\hline
\end{tabular}

Table 4. The relationship of VAS between those who were informed and were not informed about prolotherapy in patients who were not administered prolotherapy $(0=$ not informed, $1=$ informed)

\begin{tabular}{|c|c|c|c|c|c|c|}
\hline & İnformed & $\mathbf{N}$ & Mean & $\begin{array}{c}\text { Std. } \\
\text { Deviation }\end{array}$ & $\begin{array}{c}\text { Std. Error } \\
\text { of Mean }\end{array}$ & p \\
\hline \multirow{2}{*}{ VAS1 } & $0^{*}$ & 17 & 72,88 & 8,623 & 2,091 & \multirow{2}{*}{0.000} \\
\cline { 2 - 6 } & $1^{*}$ & 28 & 58,54 & 13,412 & 2,535 & \\
\hline \multirow{2}{*}{ VAS2 } & $0^{*}$ & 17 & 51,00 & 11,554 & 2,802 & \multirow{2}{*}{0.214} \\
\cline { 2 - 6 } & $1^{*}$ & 28 & 45,32 & 11,735 & 2,218 & \multirow{2}{*}{0.622} \\
\hline \multirow{2}{*}{ VAS3 } & $0^{*}$ & 17 & 43,41 & 9,042 & 2,193 & \multirow{2}{*}{$1^{*}$} \\
\cline { 2 - 6 } & $1^{*}$ & 28 & 39,96 & 9,461 & 1,788 & \multirow{2}{*}{} \\
\hline
\end{tabular}


patients to whom prolotherapy was administered were statistically significant compared to those without prolotherapy administration in their follow-ups.

Plantar fascia release is a surgical treatment for patients with plantar fasciitis unaffected by conservative treatment. Stretches due to tension of long plantar ligaments significantly increased and the average normal tension may exceed $200 \%$. Since plantar fascia has a great contribution to the load-bearing capacity of foot, its release should be considered carefully and in depth [18]. In our study, multiple perforations opened by the tip of injector during prolotherapy result in partial release of plantar fascia. We may define this as partial release of percutaneous plantar fascia (Figure 1).

The author assumes that the patient's expectation of benefiting from a particular treatment is associated with improved functional outcomes when administered. It shows that patient expectations may affect the clinical outcome independent of the treatment itself. It can help explain the apparent success of some traditional and alternative therapies in trials that do not control patient expectations. The findings may also be important for the choice of treatment in the clinical setting [19].

In a similar study conducted by Yucel and his friends; ultrasonography-guided and palpation based steroid injections were compared; At the end of the 25th month, no significant difference was found between the groups [20]. Therefore, it is thought that the palpation technique is not a disadvantage.

Prior to conservative treatment, although a significant decrease in pain of patients who were explained in detail or not explained in detail on their first arrival which treatments might be administered was determined in their first control after the conservative treatment, no significant difference was found in the long-term. This may result from the fear of injection of patients or it may be considered that detailed explanation of treatment to the patient increased the patient's compliance with the conservative treatment.

The advantages of our study are that injections were administered by a single $\mathrm{MD}$, the availability of the comparative group and the close values of BMI. I think prolotherapy is effective in the treatment of plantar fasciitis since dextrose solution can be easily obtained at affordable prices, operation is percutaneous and lacks serious complications and it is effective in alleviating pain in the early stage. The disadvantages of our study: Lack of dry and steroid injection in the comparison group. It is evident that further comparative studies with larger patient groups are needed to evaluate the efficacy of prolotherapy.

\section{Conclusion}

The exercise regime applied in this study alleviates the pain associated with chronic plantar facitiis. However, prolotherapy resulted in a decrease in pain in the early period compared to the conservative treatment. Pre-treatment (prolotherapy) VAS scores of patients who were informed about what would be done in the post-treatment were lower than the patients who were not informed.

\section{References}

1. Neufeld SK, Cerrato R (2008) Plantar fasciitis: evaluation and treatment. J Am Acad Orthop Surg 16: 338-346. [Crossref]

2. Weiss LD (2007) Easy injections. Elsevier Health Sciences.

3. Rabago D, Slattengren A, Zgierska A (2010) Prolotherapy in primary care practice. Prim Care 37: 65-80. [Crossref]

4. Roditi D, Robinson ME (2011) The role of psychological interventions in the management of patients with chronic pain. Psychol Res Behav Manag 4: 41-49. [Crossref]

5. Cotchett MP, Landorf KB, Munteanu SE (2010) Effectiveness of dry needling and injections of myofascial trigger points as $\neg$ sociated with plantar heel pain: a systematic review. J Foot Ankle Res 3: 18. [Crossref]

6. Othman AMA, Ragab EM (2010) Endoscopic plantar fasciotomy versus extracorporeal shock wave therapy for treatment of chronic plantar fasciitis. Arch Orthop Trauma Surg 130: 1343-1347. [Crossref]

7. Lim AT, How CH, Tan B (2016) Management of plantar fasciitis in the outpatient setting. Singapore Med J 57: 168-171. [Crossref]

8. Lynch DM, Goforth WP, Martin JE, Odom RD, Preece CK, et al. (1998) Conservative treatment of plantar fasciitis. A prospective study. J Am Podiatr Med Assoc 88: 375380. [Crossref]

9. Schwartz EN, Su J (2014) Plantar Fasciitis:A Concise Review. Perm J 18: 105-107. [Crossref]

10. Li S, Shen T, Liang Y, Zhang Y, Bai B (2014) Miniscalpel-Needle versus Steroid Injection for Plantar Fasciitis: A Randomized Controlled Trial with a 12-Month FollowUp. Evid Based Complement Alternat Med 14: 164714 [Crossref]

11. Tiwari M, Bhargava R (2013) Platelet rich plasma therapy: A comparative effective therapy with promising results in plantar fasciitis. J Clin Orthop Trauma 4: 31-35. [Crossref]

12. Van Pelt RS (2011) Ankle and Foot Treatment with Prolotherapy. J of Prolotherapy 3: $576-581$.

13. Rivello GJ, Hajimirsadheghi AN (2015) Clinical Effects of Pro-lotherapy for Chronic Foot and Ankle Pain. The Podiatry Institute 35: 181-186.

14. Sanderson LM, Bryant A (2015) Effectiveness and safety of prolotherapy injections for management of lower limb tendinopathy and fasciopathy: a systematic review. $J$ Foot and Ankle Res 8: 57. [Crossref]

15. Szabó G, Marcsik A, Farkas C (2010) Results of patient information and of a therapeutic exercise program in the treatment of plantar fasciitis. Orv Hetil 151: 698701. [Crossref]

16. Apaydın AH, Örsçelik A, Yıldız Y (2018) Plantar Fasiitli Rekreasyonel Sporcularda Proloterapi Uygulamasının Etkinliği. Spor Hekimliği Dergisi 53: 037-046.

17. Hauser RA, Lackner JB, Steilen-Matias D, Harris DK (2016) A Systematic Review of Dextrose Prolotherapy for Chronic Musculoskeletal Pain. Clin Med Insights Arthritis Musculoskelet Disord 9: 139-159. [Crossref]

18. Gefen A (2002) Stress analysis of the standing foot following surgical plantar fascia release. J Biomech 35: 629-637. [Crossref]

19. Kalauokalani D, Cherkin DC, Sherman KJ, Koepsell TD, Deyo RA (2001) Lessons from a trial of acupuncture and massage for low back pain: patient expectations and treatment effects. Spine 26: 1418-1424. [Crossref]

20. Yucel I, Yazici B, Degirmenci E, Erdogmus B, Dogan S (2009) Comparison of ultrasound, palpation and scintigraphy-guided steroid injections in the treatment of plantar fasciitis. Arch Orthop Trauma Surg 129: 695-701. [Crossref]

Copyright: (C2019 Taşdemir Z. This is an open-access article distributed under the terms of the Creative Commons Attribution License, which permits unrestricted use, distribution, and reproduction in any medium, provided the original author and source are credited. 